Proceedings

\title{
OMICs Role in Hereditarian Prostate Cancer ${ }^{\dagger}$
}

\author{
Sergio Cuenca-Lopez 1,*, Patricia Maria Porras-Quesada ${ }^{2}$, Fernando Vazquez-Alonso ${ }^{3}$, Victor Sanchez-Conde ${ }^{3}$, \\ Maria del Pilar Gomez-Matas ${ }^{3}$, Adoracion Aneas-Alaminos ${ }^{3}$, Veronica Arenas-Rodriguez ${ }^{1}$, \\ Blanca Cano-Gutierrez ${ }^{3}$, Luis Javier Martinez-Gonzalez ${ }^{1}$ and Maria Jesus Alvarez-Cubero ${ }^{1,2, *}$
}

1 GENYO (Centre for Genomics and Oncological Research, Pfizer-University of Granada-Andalusian Government), Av. de la Ilustracion, 114, 18016 Granada, Spain; veronica.arenas@genyo.es (V.A.-R.); luisjavier.martinez@genyo.es (L.J.M.-G.)

2 Department of Biochemistry and Molecular Biology III and Immunology, Faculty of Medicine, University of Granada, Av. de la Investigacion, 11, 18016 Granada, Spain; patriciaporras@correo.ugr.es

3 Andalusian Health Service, Andalusian Government, 18004 Granada, Spain; fvazquezalonso@gmail.com (F.V.-A.); vaitor1991@gmail.com (V.S.-C.); macasarmpgm@gmail.com (M.d.P.G.-M.); dorienfermera@gmail.com (A.A.-A.); blancacanoguti@gmail.com (B.C.-G.)

* Correspondence: scuenca@ugr.es (S.C.-L.); mjesusac@ugr.es (M.J.A.-C.)

+ Presented at the First International Electronic Conference on Genes: Theoretical and Applied Genomics, 2-30 November 2020; Available online: https://iecge.sciforum.net/.

Citation: Cuenca-Lopez, S.; PorrasQuesada, P.M.; Vazquez-Alonso, F.; Sanchez-Conde, V.; Gomez-Matas, M.d.P.; Aneas-Alaminos, A.; ArenasRodriguez, V.; Cano-Gutierrez, B.; Martinez-Gonzalez, L.J.; AlvarezCubero, M.J. OMICs Role in Hereditarian Prostate Cancer. Proceedings 2021, 76, 5. https://doi.org/ 10.3390/IECGE-07147

Published: 2 November 2020

Publisher's Note: MDPI stays neutral with regard to jurisdictional claims in published maps and institutional affiliations.

Copyright: $\odot 2020$ by the authors. Licensee MDPI, Basel, Switzerland. This article is an open access article distributed under the terms and conditions of the Creative Commons Attribution (CC BY) license (http://creativecommons.org/licenses/by/4.0/).

\begin{abstract}
Prostate cancer (PC) is one of the most prevalent tumours in the world, however, the hereditary (Hereditary PC; HPC) form is a rare pathology, that does not exceed $6 \%$. Despite its very low incidence, a family history of PC in a first-degree relative multiplies the risk of suffering from PC by approximately two-fold. Therefore, the search for genetic variables associated with the detection, monitoring and treatment of the condition is paramount. In this study, we conduct deep screening of exomes by next-generation sequencing (NGS) analysis in search of new biomarkers. We performed this analysis in a family with a high incidence of PC. Our data reveal that variants in some genes, such as HIBCH and DPP4, are present in all HPC patients. Moreover, high-risk patients have unique additional variants, such as FANK1, TUBA3FP and ALDH3B2. These results provide a new set of promising biomarkers in HCP.
\end{abstract}

Keywords: hereditary prostate cancer; OMICs technologies; next-generation sequencing; biomarker

\section{Introduction}

Although all men are at increased risk of prostate cancer (PC)-which becomes greater with increasing age-a family history of PC in a first-degree relative multiplies that risk by approximately two-fold. Most cases of PC occur sporadically in people with no family history of the condition. However, approximately 5 to $10 \%$ of PC cases are believed to be primarily caused by a genetic predisposition to the condition [1]. Around 43\% of men with a diagnosis of PC before the age of 55 have "hereditary" PC (HPC). HPC refers to a specific subtype of "familial" PC. The criteria for HPC is having a family with three generations affected, three first-degree relatives affected, or two relatives affected before age 55 [2].

To improve the treatment of PC it is necessary to better understand the molecular pathways involved, as well as the regulation of these pathways in HPC. Thanks to NGS (next-generation sequencing technology), great advances in the knowledge of this tumour have been obtained [3]. Nevertheless, currently there are scarce data regarding large family cohorts, limiting progress in finding out more details related to HPC malignancy. It is crucial to obtain more information about the molecular basis of this disease in order to break down the alterations in the metabolic pathways of these families and discover how these alterations could influence the appearance and evolution of this tumour in sporadic cases. 
NGS has proven to be a significant tool for finding new altered genes, allowing for the diagnosis of many diseases, with an especially important role in less well-known diseases, such as HPC. Moreover, NGS helps expand our knowledge of control mechanisms of genetic expression with a high level of detail and accuracy, which is important for the comprehension of genotype-phenotype relationships among patients [4]. The implementation of "omics" data in clinical practice has generated a new framework in the study of health and the causes of disease; including new knowledge and approaches related to precision medicine. Thanks to all these methodologies it is reasonable to expect extraordinary progress in our knowledge of the genome, its expression and regulation related to the molecular mechanisms associated with health.

Here, we provide a deep analysis of exome data, analysed by NGS technology, in order to understand the role of new reported variants in contributing to the aggressiveness of variants of this tumour.

\section{Materials and Methods}

\subsection{Samples}

We performed an analysis in a family of a high incidence of PC (family pedigree details in Figure 1). Blood samples were collected in EDTA and Tempus ${ }^{\mathrm{TM}}$ tubes and processed $24 \mathrm{~h}$ before collection by separation from plasma by centrifugation at $120 \mathrm{~g}$ for 20 min. Samples were then stored at $-80^{\circ} \mathrm{C}$ until they being processed. In this family, the mean tumour detection age was around 50 years old, with prostate-specific antigen (PSA) values between 7 to $12 \mathrm{ng} / \mathrm{mL}$, a Gleason score of eight and a TNM classification of around two or three. Samples were collected in 2017, so data regarding the follow-up and quality of life of patients are available. All study participants provided written informed consent before being enrolled, and the study was previously approved by the Research Ethics Committee of Granada Centre (CEI-Granada internal code 0166-N-19) following the Helsinki ethical declaration.
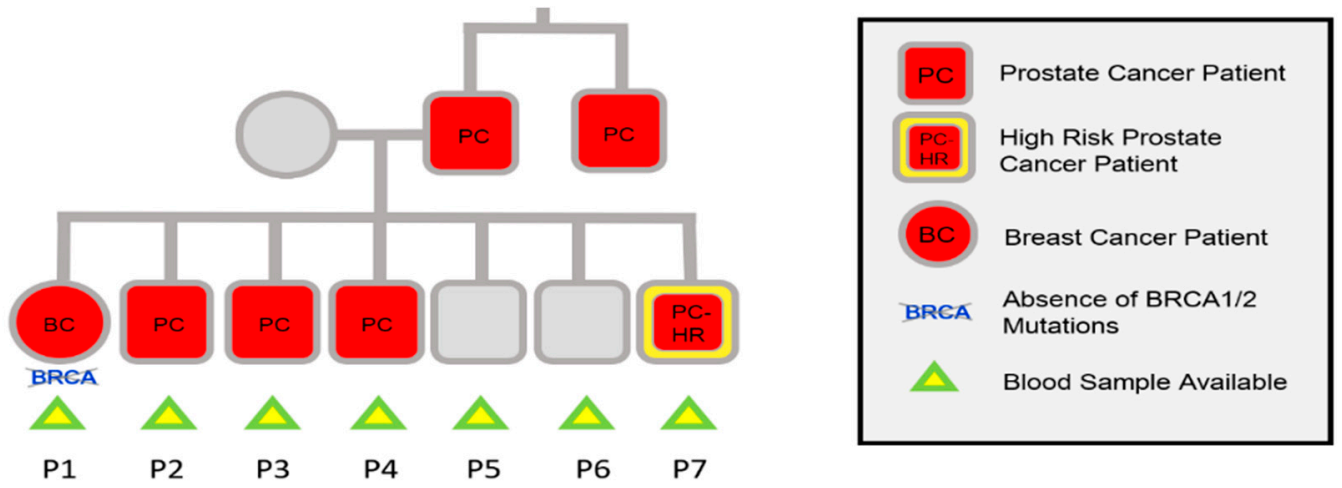

Figure 1. Family pedigree representation.

\subsection{Next-Generation Whole-Exome Sequencing (WES) for Candidate Gene Identification}

DNA were extracted using kit "DNA Blood Mini" (Qiagen Hilden, Germany) with automatized extraction using Qiacube (Qiagen Hilden, Germany). For preparing the library, $0.5 \mu \mathrm{g}$ of DNA from all de samples were collected after quantification by Qubit v4 (Thermo Fisher Scientific, MA, USA). DNA from all samples was fragmented using Covaris E200 (Covaris, MA, USA) following the manufacturer's recommendations. "Hybridization-Based Target Enrichment" by Nimblegen Roche was used for exome capture, using reagents "HyperCapWorkflow v2.0" (Roche Sequencing Solutions, CA, USA) and "SeqCap ${ }^{\circledR}$ EZ MedExome" (Nimblegen Roche, Basel, Switzerland). Libraries were then sequenced by NextSeq 500 (Illumina, CA, USA) using the kit NextSeq 500/550 High Output Kit v2.5 (300Cycles) for producing paired sequences $150 \times 2$. 


\subsection{Bioinformatic Analysis}

To find significant genetic variations within the family of high-risk PC, various treatments were carried out on the sequencing data obtained. The most significant genetic variations, according to the parameters provided by the SIFT [5] and PolyPhen [6] predictors, were selected. Among the selected variants, those that met two specific criteria were chosen. The first criteria included those variants that are expressed only in PC patients and not in healthy patients, in other words, variants expressed in patients P2, P3, P4 and P7, and not in P1, P5 and P6 (code assigned to each patient shown in Figure 1). On the other hand, the variants found only in patients with high-risk PC (most aggressive form of the disease), P7, were included in the second pattern.

\section{Results}

To investigate the aetiology and help in understanding this disease, exome sequencing was performed in the seven siblings belonging to the study family. Sequencing analyses revealed (i) the presence of variations in five genes for only the patients with PC (P2, P3, P4 and P7): HIBCH, DPP4, MOK PPP4R3A and STK31. All of them, except STK31, were also found in the patient with breast cancer. (ii) The patient with aggressive PC (P7) showed a total of ten genes with unique variations not present in the other patients: $A C E$, ALDH3B2, CD63, EFCAB13, FAM86HP, FANK1, MYO15B, SCL25A5, TUBA3FP and ZNF142. All genes and their associated single nucleotide polymorphisms (SNPs) are shown in Table 1.

Table 1. List of variations obtained after bioinformatics analysis in prostate cancer (PC) patients.

\begin{tabular}{|c|c|}
\hline \multicolumn{2}{|c|}{ Variations in All PC Patients } \\
\hline Gene & SNP \\
\hline$D P P 4$ & rs116302758 \\
\hline $\mathrm{HIBCH}$ & rs291466 \\
\hline MOK & rs56377169 \\
\hline \multicolumn{2}{|l|}{ PPP4R3A } \\
\hline \multicolumn{2}{|l|}{ STK31 } \\
\hline \multicolumn{2}{|c|}{ Variations in High Risk PC Patient } \\
\hline Gene & SNP \\
\hline \multicolumn{2}{|l|}{$A C E$} \\
\hline ALDH3B2 & rs7947754 \\
\hline \multicolumn{2}{|l|}{ CD63 } \\
\hline EFCAB13 & rs2271803 \\
\hline FAM86HP & rs16834628 \\
\hline \multicolumn{2}{|l|}{ FANK1 } \\
\hline MYO15B & rs73998360 \\
\hline \multicolumn{2}{|l|}{ SLC25A5 } \\
\hline TUBA3FP & rs2075276 \\
\hline ZNF142 & \\
\hline
\end{tabular}

\section{Discussion}

In the current study, we showed how genetic variation correlates with PC, depending on being in a healthy or affected family (increasing in different aggressiveness stages). Whole-exome sequencing was performed to identify variants within the coding region of the genome that were associated with HPC aggressiveness. The aggressiveness of the disease was monitored by Gleason score, PSA levels and the quality of life of patients. The death of a high-risk PC patient (P7) in this study has been recently reported. 
Our data support the notion that several genes, such as $H I B C H, D P P 4, M O K$ and PPP4R3A, are present only in HPC patients. Although the data are scarce, previous publications have related $\mathrm{HIBCH}$ with PC [7], in addition to accelerating PC progression following androgen deprivation therapy in DPP4 [8]. These data support our results and suggest that these genes could be promising biomarkers for aggressiveness, mainly due to the bad response of therapy, which has also been confirmed for other tumours, such as colorectal cancer in $\mathrm{HIBCH}$ [9]. Furthermore, this is the first time that PPP4R3A and MOK have been related to PC. PPP $4 R 3 A$ has some related pathways; it is included breast cancer and glucagon signalling [10]. A carcinogenesis event could be explained by its role during DNA replication and DNA double-strand break repair. Additionally, MOK belongs to the $M A P$ kinase superfamily and its related pathways includes IL-2 [10]. Moreover, high-risk patients have unique additional variants, such as $A C E, C D 63$ and EFCAB13, that have already been associated with $P C$. These results provide a new set of promising biomarkers that can help in the stratification and diagnosis of HCP.

Funding: This research is part of the project "Integration of omics data in multi-case families with prostate cancer for application in liquid biopsy (BIOOMIC-CP)" which was funded by Ministerio de Ciencia, Innovación y Universidades (MINECO) (Spanish Government Institution). PID2019110512RA-I00, 01/06/2020-01/07/2023.

Institutional Review Board Statement: The study was conducted according to the guidelines of the Declaration of Helsinki, and approved by Research Ethics Committee of Granada Centre (CEI-Granada, internal code 0166-N-19, 05/03/2019).

Informed Consent Statement: Informed consent was obtained from all subjects involved in the study.

Data Availability Statement: The data presented in this study are available on request from the corresponding author. The data are not publicly available due to ethical issues.

Conflicts of Interest: The authors declare no conflict of interest.

\section{References}

1. PDQ Cancer Genetics Editorial Board. Genetics of Prostate Cancer (PDQ $\left.{ }^{\circledR}\right)$ : Health Professional Version; PDQ Cancer Genetics Editorial Board: Bethesda, MD, USA, 2002.

2. Potter, S.R.; Partin, A.W. Hereditary and familial prostate cancer: Biologic aggressiveness and recurrence. Rev. Urol. 2000, 2, 3536.

3. van Dessel, L.F.; van Riet, J.; Smits, M.; Zhu, Y.; Hamberg, P.; van der Heijden, M.S.; Bergman, A.M.; van Oort, I.M.; de Wit, R.; Voest, E.E.; et al. The genomic landscape of metastatic castration-resistant prostate cancers reveals multiple distinct genotypes with potential clinical impact. Nat. Commun. 2019, 10, 1-13.

4. Martinez-Gonzalez, L.J.; Antúnez-Rodríguez, A.; Vazquez-Alonso, F.; Hernandez, A.F.; Alvarez-Cubero, M.J. Genetic variants in xenobiotic detoxification enzymes, antioxidant defenses and hormonal pathways as biomarkers of susceptibility to prostate cancer. Sci. Total Environ. 2020, 730, 138314.

5. Vaser, R.; Adusumalli, S.; Ngak Leng, S.; Sikic, M.; Ng, P.C. SIFT missense predictions for genomes. Nat. Protoc. $2015,11,1$.

6. Adzhubei, I.A.; Schmidt, S.; Peshkin, L.; Ramensky, V.E.; Gerasimova, A.; Bork, P.; Kondrashov, A.S.; Sunyaev, S.R. A method and server for predicting damaging missense mutations. Nat. Methods 2010, 7, 248-249.

7. Graff, J.N.; Puri, S.; Bifulco, C.B.; Fox, B.A.; Beer, T.M. Sustained complete response to CTLA-4 blockade in a patient with metastatic, castration-resistant prostate cancer. Cancer Immunol. Res. 2014, 2, 399-403.

8. Russo, J.W.; Gao, C.; Bhasin, S.S.; Voznesensky, O.S.; Calagua, C.; Arai, S.; Nelson, P.S.; Montgomery, B.; Mostaghel, E.A.; Corey, E.; et al. Downregulation of dipeptidyl peptidase 4 accelerates progression to castration-resistant prostate cancer. Cancer Res. 2018, 78, 6354-6362.

9. Shan, Y.; Gao, Y.; Jin, W.; Fan, M.; Wang, Y.; Gu, Y.; Shan, C.; Sun, L.; Li, X.; Yu, B.; et al. Targeting HIBCH to reprogram valine metabolism for the treatment of colorectal cancer. Cell Death Dis. 2019, 10, 1-15.

10. Stelzer, G.; Rosen, N.; Plaschkes, I.; Zimmerman, S.; Twik, M.; Fishilevich, S.; Iny Stein, T.; Nudel, R.; Lieder, I.; Mazor, Y.; et al. The GeneCards suite: From gene data mining to disease genome sequence analyses. Curr. Protoc. Bioinform. 2016, 54, 1.30.11.30.33. 